\title{
Environment and structure influence on DNA conduction
}

\author{
Ch. Adessi, S. Walch and M.P. Anantram \\ NASA Ames Research Center, \\ Mail Stop: Ni29-3, \\ Moffett Field, CA 94035-1000, USA
}

(Dated: September 24, 2002)

\begin{abstract}
:
Results for transmission through the poly $\{G\} D N \&$ molecule are presented. We show that (i) periodically arranged sodium counter-ions in close proximity to dry DNA gives rise to a new conduction channel and aperiodicity in the counter-ion sequence can lead to a significant reduction in conduction, (ii) modification of the rise of B-DNA indtces a change in the width of the transmission window, and (iii) specifically designed sequences are predicted to show intrinsic resonant tunneling behavior.

PACS numbers: 72.10.-d, 85.65.+h, 87.15.-v, 73.22.-f
\end{abstract}


The growing interest in DNA for nanotechnology and molecular devices stems from the ability of controlled growth of nucleotide sequences and its surprising conducting properties. Recent transport measurements on DNA strands lying between metallic electrodes have produced contradictory results. Fink et al. ${ }^{1}$ observed metallic behavior in $\lambda$ DNA using an electron projection microscope set-up, Kasumov et al. ${ }^{2}$ observed superconducting behavior, Porath et $a .^{3}{ }^{3}$ observed semiconducting behavior with a well defined poly $\{\mathrm{G}\}$-poly $\{\mathrm{C}\} \mathrm{DNA}$ molecule, and de Pablo el $a l^{4}{ }^{4}$ observed insulating behavior using a scanning force microscope based set up. Biochemists have established a link between oxidative damage of DNA due to hole transport and the occurrence of genetic mutations. It has also been shown that oxidative damage is sequence dependent. ${ }^{5}$ Experiments $^{6,7}$ and modeling ${ }^{8,9}$ have shown that both short range coherent tunneling transport and long range incoherent / hopping transport ${ }^{8-10}$ are mechanisms for hole transport.

Charge transport in DNA is a complex phecomenon because the environment of the DNA plays a significant role in determining "he energy levels of nucleotides and the structure. ${ }^{11}$ Transformation of DNA structure has been correlated to the $\mathrm{pH}$ of the buffer. In this letter, we model some aspects of DNA structure and environment that influence charge transport.

Model and Method: The system modeled is a single strand B DNA molecule, which has ten bases per turn with a distance of $3.38 \AA$ between consecutive bases. We considered two cases. In case $\mathrm{A}$, only the nucleic acids are included with an $\mathrm{H}$ atom termination of the base. For two bases there are six degrees of freedom which lead to a very large number of possible conformations. We calculate the change in transmission probability through an infinite poly $\{\mathrm{G}\}$ DNA molecule due to change ir two of these degrees of freedom: rise (the distance between bases) and twist (angle between consecutive bases). The coordinates of the molecule are generated by applying the translations and rotations given in Ref. 12 to a single nucleotide. In case $\mathrm{B}$, the sugar phosphat 3 backbone and nucleic acids are explicitly included with the structure taken from Ref. 12. Hydration shells are absent in our model, and fluctuations in DNA conformation do not exist as in the case of dry DNA. We consider a straight forward B DNA model because the st:ucture of DNA lying on a substrate is not well understood. ${ }^{13}$ In the calculations involving the effect of the counter-ions (cations) of the phosphate group on charge transport, the backlione and counter-ions are both considered. 
The location of the counter-ions are determined $b y$ energy minimization ${ }^{14}$ of a one phosphate two sugar model of the DNA backbone.

The transmission through the molecule is computed using a Green's function framework. ${ }^{15}$ More specifically, the method used follows Ref. 16, which is based on considering only the block tridiagonal matrix representation of the Hamiltonian to reduce numerical complexity. The diagonal blocks of the Hamiltonian represent the Hamiltonian of the bases, and the off-diagonal blocks represent interaction ketween nearest neighbor bases. Interaction with bases that are not immediate neighbors along the strand are neglected. In constructing the block tridiagonal Hamiltonian, we follow the work of Gadzy et al. ${ }^{17}$ who gave a prescription to construct the block tridiagonal Hamiltonian for an extended system based on the Hamiltonian obtained from calculations on dimers or trimers. Such a calculation has been carried out in the context of the DNA by Ye and Jiang. ${ }^{18}$ We use the methods of ref. 18 to construct the block tridiagonal blocks and ref. 15 to compute the transmission and density of states. Our calculations on dimers, along the DNA chain to obtain the Hamiltonian / Fock matrix ${ }^{18}$ are performed using the B3LYP ex hange-correlation functional ${ }^{19,20}$ in density functional calculations using Gaussian 98. ${ }^{14}$ Calculations of long DNA chains are based on the Fock matrices of the dimers and further self-consistency is neglected. The basis set used here corresponds to 6-21G. More diffuse or complete basis sets affect our results little.

The transmission through the molecule is computed using the following expression:

$$
T(E)=\operatorname{tr}\left(\Gamma_{L} C_{r}^{r} \Gamma_{R} G^{a}\right)
$$

In this equation, $G^{r(a)}$ represents the retarded (advanced) Green's function and $\Gamma_{L(R)}$ the coupling between the left (right) lead and the device. $G^{r}$ is the solution of the following equation:

$$
\left(E S-H-\Sigma_{L}^{r}-\Sigma_{R}^{r}\right) G^{r}=\mathbb{1},
$$

where $E$ represents the energy of the electron, $H$ the Hamiltonian, $S$ the overlap matrix and $\Sigma_{L(R)}$ the self-energy. In the frame-work of nearest neighbor approximation, $\Gamma_{L(R)}$ and $\Sigma_{L(R)}$ are given by:

$$
\left\{\begin{array}{l}
\Sigma_{L(R)}^{r}=H_{21} g_{L(R)}^{r} H_{12}, \\
\Gamma_{L(R)}=2 \pi H_{21} \operatorname{In}\left[g_{L(R)}^{r}\right] H_{12}
\end{array}\right.
$$

where $g_{L(R)}^{r}$ is the retarded surface Green's function of the left (right) lead, and $\operatorname{Im}[x]$ represents the imaginary part of $x$. 
Results: The highest occupied molecular orbital (HOMO) and the lowest unoccupied molecular orbital (LUMO) of a nucleotide primarily lie on the bases. Hence it is believed that transport in native DNA occurs primarily along the base pairs, and the backbone does not play a role apart from providing mechanical stability to the structure. ${ }^{21}$ Fig. 1 corresponds to the variation of transmission when the rise of a B-DNA is decreased (model $\mathrm{A}$ ). When this distance is decreased from the regular value of $3.38 \AA$, the main effect is a broadening of the conduction channels and a shift of the HOMO channel. Physically, the broadening occurs due to an increase in the hybridization between the $\pi$ orbitals of the guanine. An increase of the rise (distance between consecutive bases) causes a shift and sharpening of the channel because the hybridization between the $\pi$ orbitals of neighboring bases is now diminished. Energetically, the decrease of the distance between nearest neighbor bases by $0.28 \AA$ produces an increase in the total energy of only $0.14 \mathrm{eV}$ for a two base system. Thus, even if this conformation is energetically higher, it may be encountered in specific conditions. Also, it may be possible to perforr experiments where DNA lying between contacts can be stretched as in recent experiments with carbon nanotubes. ${ }^{22}$ Broadening of the HOMO transmission window is also seen when the twist angle is decreased from its regular value of $36^{\circ}$ (Fig. 2) as recently found by Di Felice et al. in Ref. 23. In the absence of strain, the width of the HOMO transmission char nel is $370 \mathrm{meV}$ (Fig. 1), which corresponds to an average nearest neighbor hopping strength between bases of nearly $90 \mathrm{meV}$. Changing the twist angle from $36^{\circ}$ to $30^{\circ}$ and $0^{\circ}$ changes the width of the HOMO transmission channel to $270 \mathrm{meV}$ and $1.07 \mathrm{eV}$ respectively (Fig. 2).

We now consider the effect of counter-ions using a DNA strand with the backbone included (model B). One parameter which is not well characterized in most of the transport experiments on DNA is its close environment. It is only very recently that a combined theoretical and experimental study has pointed out the possible implications of the backbone with its close environment in hole transport. ${ }^{24}$ Indeed, a DNA molecule in a saline solution is surrounded by a cloud of ionic species due tc the presence of a negative charge mainly located on one of the oxygens of the phosphate group. Measurements of current by physicists involves dry DNA. In dry DNA, where are the cations and hydration shells located, and is a hydration shell necessary or can it be avoided cll together? These questions are extremely difficult to answer at this initial stage of the fielit. The answer will probably come from an interplay of high resolution STM/AFM measurements and modeling, which is well beyond 
the scope of our work. Our model study here makes simple assumptions about the location of the cations without a hydration shell, to first understand what happens in this idealistic limit. As is shown below, there are very interesting issues even in this limit. We find that the cations have two main influences on conduction:

(a) Transport could occur via a channel composed of cations. This would however necessitate a periodic arrangement of cations and is studied for a few different cations. These results do not depend significantly on the distance of the cation from the phosphate group as long as it is between 2.3 and $2.8 \AA$ (distance to the nearest oxygen) and are uniformly placed along the chain.

(b) Cations affect the on-site potential of the bases, and hence alter conductance through the bases substantially. Specifically, non uniformity in cation type could lead to a reduction in conductance through the bases. We have presented results for the decrease in transmission when a single cation is replaced or removed.

We discuss (a) and (b) now. We compare the influence of Hydrogen, Hydronium and Sodium counter-ions on transmission of electrons through a poly $\{\mathrm{G}\}$ strand. The charge transfer from the counter-ion to the phosphate group is found using Mulliken population analysis to be $0.42,0.80$ and 0.60 for the cases of $\mathrm{H}, \mathrm{H}_{3} \mathrm{O}$ and $\mathrm{Na}$ respectively. So the Sodium atom has $10+0.40$ electrons and its electronic properties are different from a fully ionized Sodium atom with only ten electrons, which wo lld be akin to Neon. In the case of $H$ and $\mathrm{H}_{3} \mathrm{O}$, we find that the HOMO and LUMO chantels are still on the base and so conduction would occur primarily through the base. In the calculations with $\mathrm{Na}$, we find that new conduction channels occur in the HOMO-LUMO gap (Potassium and Lithium also show this feature but is not discussed here). Fig. 3 shows the plot of transmission versus energy with $\mathrm{Na}$ counter-ions. The local density of states at the Sodium atom (not shown) further prove that the new channel (located at $-2.6 \mathrm{eV}$ ) is due to the sodium atoms. That is, the phosphate group acts as a template for a helical wire consisting of Sodium atoms, conduction through which gives rise to the new channel. We have also verified that the transmission obtained by replacing the combined system of phosphate group, sugar and base by $\mathrm{OH}$ at the location of the phosphate groups leads to the occurrence of these conduction channels due to the Sodium wire. The above results indizate that depending on the location of the Fermi energy, the counter-ions of the phosphate group could play a role in determining the conductance. Of course, as already mentioned, this necessitates uniformly arranged 
counter-ions along the DNA strand.

We now present some results for the influence of more than one ionic species in the backbone. Experimentally, when DNA dries, we expect that there would be variation in both the type and location of the counter-ion. We consider a system with a chain of Sodium counter-ions, where a single Sodium atom is replaced by $\mathrm{H}_{3} \mathrm{O}$. The main physical effect of the $\mathrm{H}_{3} \mathrm{O}$ counter-ion is to shift the HOMO and LUMO of the base closest to it, relative to other bases with a Sodium counter-ion. The transmission probability through such a system is shown in Fig. 3 (dashed line). The decrease in transmission over relevant energy ranges near the HOMO and LUMO is significant. As a result, we expect the conductance of a DNA strand with fluctuation in the type of counter-ions to be significantly altered compared to a strand with uniformly placed counter-ions. We also find that the shift in the energy levels due to $\mathrm{H}_{3} \mathrm{O}$ (with respect to Sodium) is around $150 \mathrm{meV}$, which is comparable to the hybridization energy between nearest neighbor guanines. Thus, a random sequence of counter-ions along the backbone would eventually tend to localize the electron wave function along the bases.

The principle of complementarity between bases and the ability to design arbitrary sequences has generated interest in DNA based electronics. Inspired by this, we have studied the possibility of intrinsic resonant tunneling ir. DNA strands. The underlying structure in resonant tunneling is a quantum well regicn separated by two barriers. Motivated by the fact that guanine has a lower ionization energy than thymine, we consider the following two strands which are seemingly symrnetric, poly\{G\}TGGGGGGTpoly\{G\} and poly $\{G\} T G G G T$ poly $\{G\}$. We expect the thymines to comprise a barrier and the guanines in between to form a quantum well. Three and six clear transmission resonances due to hybridization of the guanines are seen in Fig. 5 . The transmission probability through a system with symmetric barriers should peak at unity. Interestingly, the barriers created by the thymines have a small asymmetry because the overlap Hamiltonians between $G$ and $\mathrm{T}$ in $\left\{5^{\prime} \mathrm{GT}^{\prime}\right\}$ and $\left\{5^{\prime} \mathrm{TG} 3^{\prime}\right\}$ are not the same (these structures are not geometrically identical due to rotation of bases along the DNA helix). However, as the asymmetry is mild, the transmission is close to unity. Next, we consider a poly $\{G\} T T G G G T$ poly $\{G\}$ strand, which has a much larger asymmetry in the barr ers created by the thymines. Here, we find that while the location of the transmission res onances has not changed significantly, the peak values are smaller than 0.2 (inset of Fig. 4). It is noted that such resonant tunneling 
behavior may be observable in an experiment where the Fermi energy through the DNA is modified by applying a gate voltage or in experiments looking at current as a function of the applied bias between the source and drain terminals. As sequences such as those used above and transport measurements are bcth experimentally feasible, observation of resonant tunneling in DNA may be possible in the near future.

We now briefly discuss two aspects that have been ignored in our paper, the location of the Fermi level and electrostatics. Reliably computing the Fermi level of molecules connected to electrodes has proved to be a challenging prcblem because charge transfer between the macroscopic contacts and the molecules should be: precisely determined. For a large molecule such as the DNA, it might be best to use values that can be infered from experiments. Refs. 3 and 4 find that the Fermi energy lies in the FOMO-LUMO gap, which is in agreement with the expectation that a large molecule would tend to be more or less charge neutral at equilibrium. A recent interesting experimen ${ }^{25}$ has made further progress by showing that the Fermi energy in poly $\{\mathrm{G}\}$-poly $\{\mathrm{C}\}$ and foly $\{\mathrm{A}\}$-poly $\{\mathrm{T}\}$ molecules lie closer to the HOMO (p-doped) and LUMO (n-doped) chann als respectively. Experiments that involve an electrostatically coupled gate electrode have a significant advantage because the Fermi energy can be tuned from the HOMO band to the LUMO band in a controlled manner.

Our assumption of neglecting self-consistency for long strands is strictly not correct in the presence of large applied biases and for aperiodic systems. The potential profile in these scenarios will be determined by long range charge transfer. In our calculations, charge transfer is accurately accounted for only in the Fock mat ix of the subsystem. We have verified that expanding the subsystem from two to three bases does not significantly affect our results. Self-consistent calculations to determine the fraction of voltage drop across the DNA and contacts are important in understanding the mechanism of current flow at finite voltage biases but is beyond the scope of this paper. It might be of interest to note that self-consistent calculations in semiconducting carbon nanotubes: (comparable diameter to DNA) show that a larger fraction of the applied voltage drops acrcss the nanotube rather than the contacts. ${ }^{26}$

In conclusion, we have used a Green's function formalism to study the transmission properties of poly $\{\mathrm{G}\}$ DNA molecules. Decrease of the distance between the bases from its regular value for B-DNA leads to a shift and change in width of the transmission 
window. Some ionic species such as sodium could create new conduction channels. More importantly, we have shown that variation of jonic species in the backbone can cause a significant reduction in transmission through tha bases. Finally, we explore the possibility of resonant tunneling in engineered DNA structures and find that it may be possible to observe intrinsic resonant tunneling in a system composed of guanine and thymine.

This work was supported by Washington State University (Mohamed Osman) under the NASA cooperative agreement No. NCC2-5407. MPA acknowledges a Directors Discretionary Fund and useful discussions with T. R. Govindan, Chris Henze, Alexei Svizhenko (NASA Ames) and Lalitha Subramanian (Carnegie Insitute of Washington). SPW was supported by NASA contract NAS2-99092 to ELORET.

1 H.-W. Fink and C. Schönenberger, Nature 398, 407 (1999).

2 A. Kasumov, M. Kociak, S. Guéron, B. Reulet, V. Volkov, D. Klinov, and H. Bouchiat, Science 291, 280 (2001).

3 D. Porath, A. Bezryadin, S. de Vries, and C. Decker, Nature 403, 635 (2000).

4 P. de Pablo, F. Moreno-Herrero, J. Colchero, J. Gómez-Herrero, P. Herrero, A. Baró, P. Ordejón, J. Soler, and E. Artacho, Phys. Rev. Lett. 85, 4992 (2000).

5 T. Williams, D. Odom, and J. Barton, J. Am. Chem. Soc. 122, 9048 (2000).

6 S. Kelley and J. Barton, Science 283, 375 (1999).

7 F. Lewis, T. Wu, Y. Zhang, R. Letsinger, S. Greenfield, and M. Wasielewski, Science 277, 673 (1997).

8 Y. Berlin, A. Burin, and M. Ratner, J. Am. Chem. Soc. 123, 260 (2001).

9 J. Jortner, M. Bixon, T. Langenbacher, and M. Michel-Beyerle, Proc. Natl. Acad. Sci. USA 95, 12759 (1998).

10 B. Giese, J. Amaudrut, A.-K. Köhler, M. Spormann, and S. Wessely, Nature 412, 318 (2001).

11 F. C. Grozema, L. D. A. Siebbles, Yu. A. Berlin, and M. A. Ratner, CHEMPHYSCHEM 6, 536 (2002).

12 S. Arnott, P. C. Smith, and R. Chandrasekarin, Handbook of Biochemistry and Molecular Biology (G.D. Fasman, CRC Press, Cleveland, 1977), pp. 411-422. 
13 C. Rivetti and S. Codeluppi, Ultramicroscopy pp. 55-66 (2001).

14 M. J. Frisch, G. W. Trucks, H. B. Schlegel, G. E. Scuseria, M. A. Robb, J. R. Cheeseman, V. G. Zakrzewski, J. A. Montgomery, Jr., R. E. Stratmann, J. C. Burant, S. Dapprich, et al., Gaussian 98 (Gaussian Inc, Pittsburgh, PA, 1999).

S. Datta, Electronic transport in mesoscopic systems (Cambridge University Press, Cambridge, United Kingdom, 1995).

16 M. P. Anantram and T. R. Govindan, Phys. Rev. B 58, 4882 (1998).

17 B. Gazdy, M. Seel, and J. Ladik, Chem. Phys. 86i, 41 (1984).

18 Y. Ye and Y. Jiang, Int. J. Quan. Chem. 78, 112 (2000).

19 C. Lee, W. Yang, and R. Parr, Phys. Rev. B 37, 785 (1988).

20 A. Becke, J. Chem. Phys. 98, 5648 (1993).

21 D. Eley and D. Spivey, Trans. Faraday Soc. 58, 411 (1962).

22 T. Tombler, L. Chongwu zhou, L. Alexseyev, C. Jing Kong, C. Hongjie Dai, C. Lei Liu, C. Jayanthi, S.-Y. Meijie Tang, and S.-Y. Wu, Nature pp. 762-772 (2000).

23 R. Di Felice, A. Calzolari, E. Molinari, and A. Garbesi, Phys. Rev. B 65, 045104 (2002).

24 R. Barnett, C. Cleveland, A. Joy, U. Landman, and G. Schuster, Science 294, 567 (2001).

25 H-Y. Lee, H. Tanaka, Y. Otsuka, K-H. Yoo, J-O. Lee, and T. Kawai, Appl. Phys. Lett. 80, 1670 (2002).

26 J. O'Keeffe, and M. P. Anantram, Unpublished. 


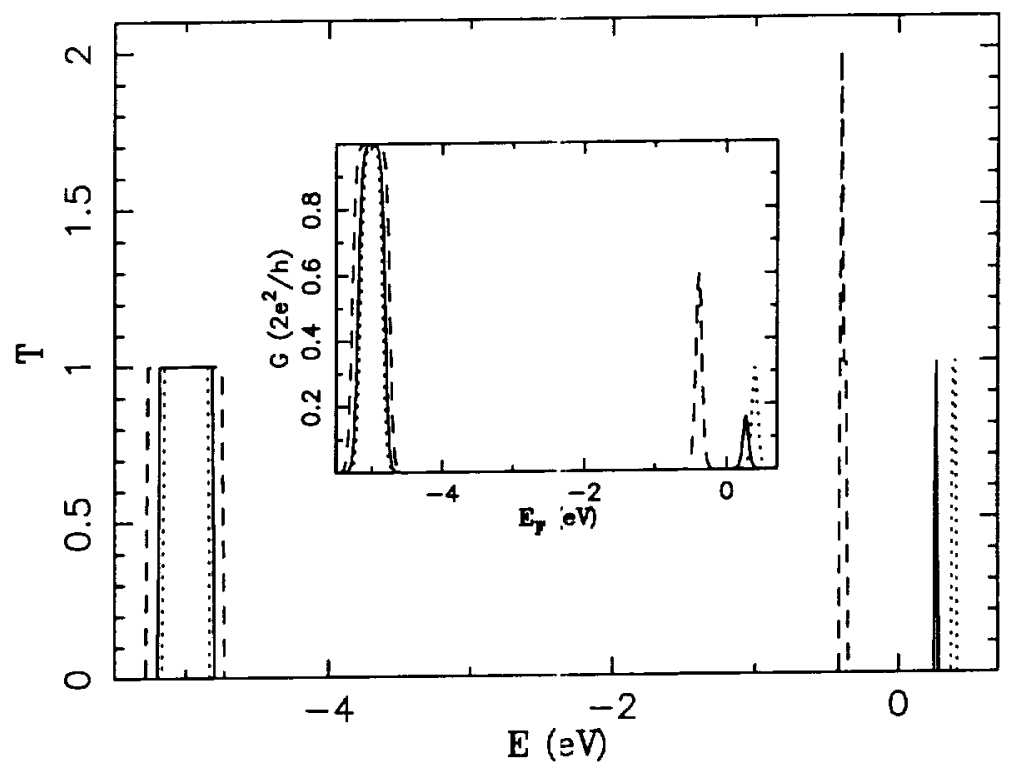

FIG. 1: Transmission versus energy for different distances between consecutive bases of a poly $\{\mathrm{G}\}$ DNA molecule without backbone. The solid line corresponds to a rise of $3.38 \AA$, the dashed line to $3.1 \AA$ and the dotted one to $3.5 \AA$. The inset corresponds to the linear response conductance at $T=298 \mathrm{~K}$.

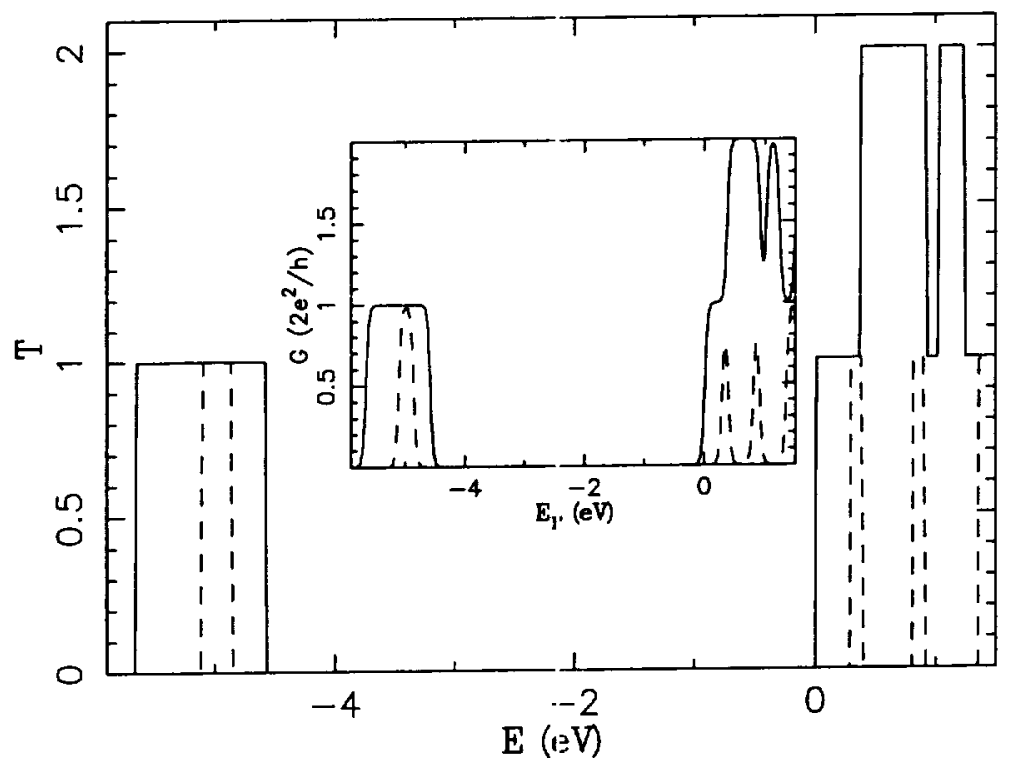

FIG. 2: Transmission versus energy for different twist angles between consecutive bases of a poly $\{\mathrm{G}\}$ DNA molecule without backbone. Solid line: $\Omega=0^{\circ}$, dashed line: $\Omega=30^{\circ}$. The inset corresponds to the linear response conductance at $T=298 \mathrm{~K}$. 


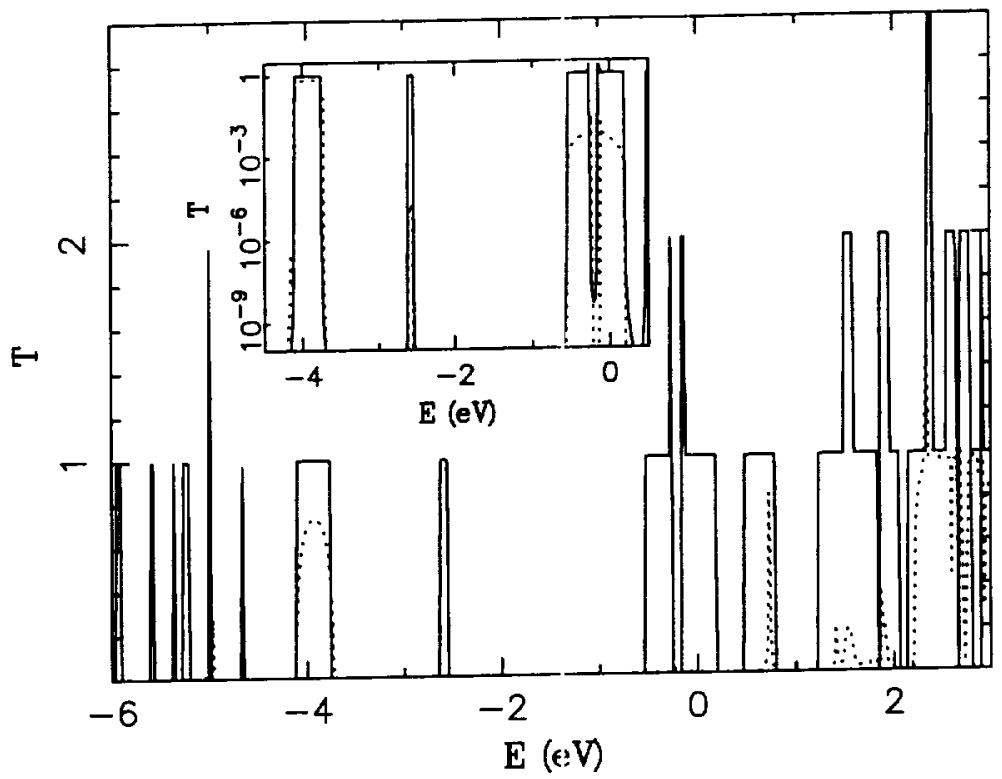

FIG. 3: Transmission versus energy for a poly $\{\mathrm{G}\}$ B-DNA surrounded by Na ions (solid line) and when a local ion mismatch is created by replacing one $\mathrm{Na}$ by a $\mathrm{H}_{3} \mathrm{O}$ (dashed line).

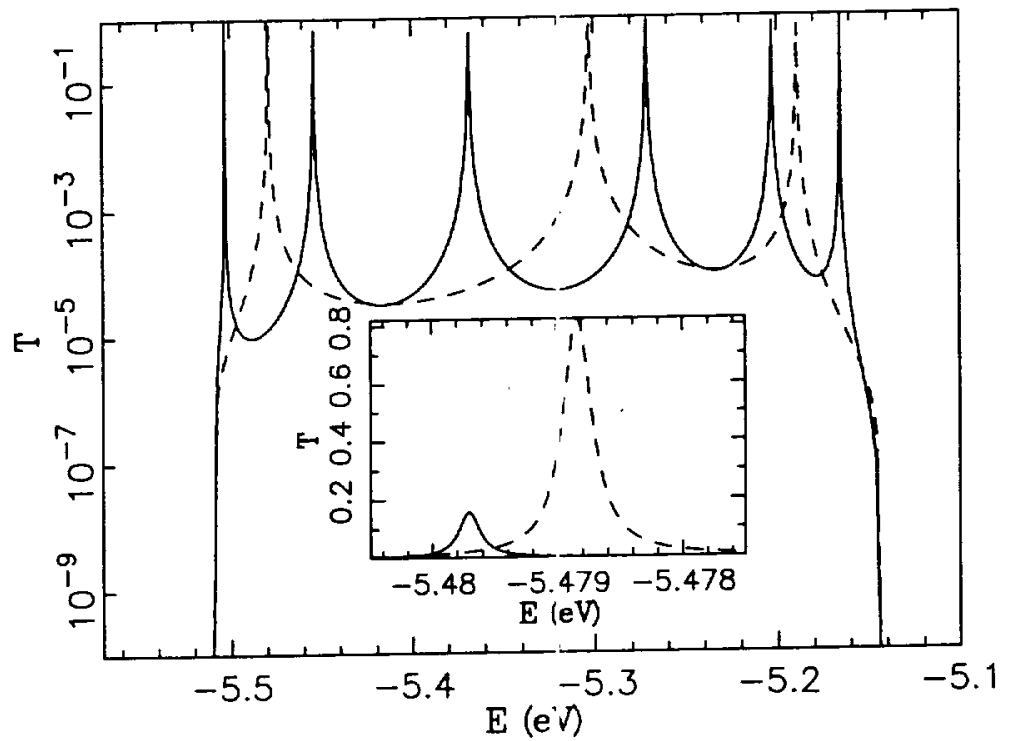

FIG. 4: Transmission versus energy for poly\{G\}TGGGTpoly\{G\} (dashed) and poly $\{$ G\}TGGGGGGTpoly\{G\} (solid). Inset: Syrnmetrical poly\{G\}TGGGTpoly\{G\} (dashed) and asymmetrical poly $\{\mathrm{G}\}$ TTGGGTpoly $\{\mathrm{G}\}$ (solid) sequences. 